“( 2014 IEEE. Personal use of this material is permitted. Permission from IEEE must be obtained for all other uses, in any current or future media, including

reprinting/republishing this material for advertising or promotional purposes, creating new collective works, for resale or redistribution to servers or lists, or reuse of any copyrighted component of this work in other works." 


\section{Message from the PUDA 2014 Workshop Chairs UIC-ATC-ScalCom 2014}

We are happy to welcome you to the Workshop on Pervasive and Ubiquitous Data Analytics (PUDA14). The workshop was held in conjunction with the 11th IEEE International Conference on Ubiquitous Intelligence and Computing (IEEE UIC-14), Ayodya Resort, Bali, Indonesia, December 9-12, 2014.

PUDA-14 contains 6 invited papers from four countries, i.e. Canada, China, Italy, and Singapore. All the papers were peer reviewed by members of the PUDA-14 program committee. The workshop addresses issues of data management in ubiquitous computing environments with a special focus on data processing. The goal of the workshop is to build a forum for researchers from academy and industry to investigate challenging and innovative research issues in the subject, which combines data analytics within pervasive and ubiquitous environment and to explore creative concepts, theories, innovative technologies and intelligent solutions, such as activity recognition, ambient context-aware search and recommendation, big data mining in mobile social networks, mobile cloud computing techniques for big data, big data protection, integrity and privacy in pervasive and ubiquitous environments, variation-aware data analytics and high-speed data stream mining, big data mining in E-education. We thank the authors for submitting their work and the members of the PUDA-14 program committee for managing the reviews of the workshop papers in such short time.

We believe this workshop contains the topics focusing on data analytics in ubiquitous environment and provides additional breadth and depth to the main conference. Finally, we hope you enjoy the workshop and have a fruitful meeting in Bali, Indonesia.

Li Liu, National University of Singapore, Singapore

Shiping Chen, CSIRO, Australia

Ching-Hsien Hsu, Chung Hua University, Taiwan

Guandong Xu, University of Technology Sydney, Australia

Xiangliang Zhang, King Abdullah University of Science and Technology, Saudi Arabia

PUDA 2014 Workshop Chairs

Li Li, Southwest University, China

Guoxin Su, National University of Singapore, Singapore

Ming Liu, Southwest University, China

Zigang Huang, Arizona State University, USA

Tingshao Zhu, Chinese Academy of Sciences, China

Jiong Jin, Swinburne University of Technology, Australia

Darren Carlson, National University of Singapore, Singapore

Weibin Chen, Huaqiao University, China

Bin Wang, Xiamen Road \& Bridge Information Engineering Co. Ltd, China

Ning An, Hefei University of Technology, China

Yi Yang, Lanzhou University, China

PUDA 2014 Program Committee 